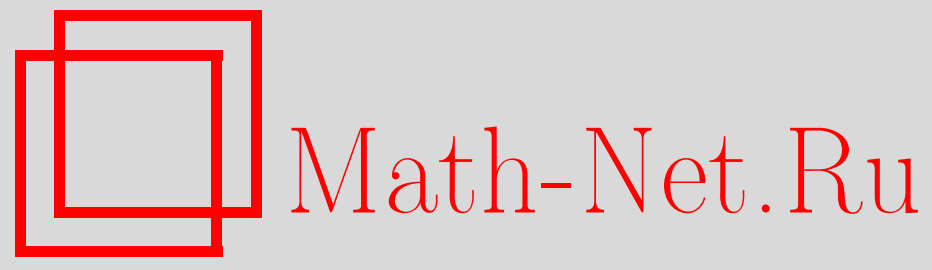

Д. И. Завершинский, В. Г. Макарян, Д. П. Порфирьев, Взаимодействие ударных волн с областью неравновесности в колебательно-возбужденном газе, Вестн. Сам. гос. техн. ун-та. Сер. Физ.-мат. науки, 2012, выпуск 3(), 203-207

DOI: https://doi.org/10.14498/vsgtu1109

Использование Общероссийского математического портала Math-Net.Ru подразумевает, что вы прочитали и согласны с пользовательским соглашением

http://www.mathnet.ru/rus/agreement

Параметры загрузки:

IP : 54.237 .59 .107

26 апреля 2023 г., $17: 46: 37$

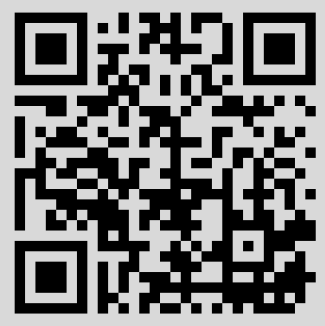




\title{
ВЗАИМОДЕЙСТВИЕ УДАРНЫХ ВОЛН С ОБЛАСТЬЮ НЕРАВНОВЕСНОСТИ В КОЛЕБАТЕЛЬНО-ВОЗБУЖДЕННОМ ГАЗЕ
}

\author{
Д. И. Завершинский В.Г. Макарян, Д. П. Порфиръев \\ Самарский государственный аэрокосмический университет им. ак. С. П. Королёва \\ (национальный исследовательский университет), \\ 443086, Россия, Самара, Московское ш., 34. \\ E-mail: vmak@rambler.ru
}

На основе численного решения уравнений газодинамики неравновесной средъ исследовано проникновение ударной волны в область неравновесного газа. Наблюдалось расщепление фронта ударной волны на ударную и тепловую волны, что качественно совпадает с экспериментальными результатами А. И. Климова.

Ключевые слова: ударная волна, неравновесность, колебателъно-возбужденный газ, ударная адиабата, тепловая волна.

Набор эффектов и явлений неравновесной газодинамики, требующих теоретической интерпретации, появился в ходе развития авиа- и космической техники последнего поколения, требующей новых подходов к аэродинамике полёта, в том числе в сильно неравновесных условиях взаимодействия с атмосферами планет. Задачи, стоящие перед гиперзвуковой авиацией пятого поколения, привели к идее управления не с помощью механических элементов, перемещаемых за счёт использования рычагов и гидравлики (что весьма затруднительно из-за большого сопротивления воздуха при гиперскоростях), а путём искусственного создания потока плазмы на крыле. Для этого, например, на передней кромке крыла можно искусственно создать плазменный разряд. При этом набегающий воздух, попадая в область электрического разряда, превращается в ионизированный газ, которым можно управлять под воздействием магнитного поля. В результате родилось новое междисциплинарное направление, получившее название плазменной аэродинамики. В этой области за последнее время проведено большое число экспериментов, показавших, что при движении тел в плазменной оболочке наблюдается снижение аэродинамического сопротивления и рост подъёмной силы при сверхзвуковом обтекании тел до $40 \%$ при наличии плазменных образований перед ними; ослабление и деструкция ударной волны перед летящими телами в плазмодинамических экспериментах, или наоборот, усиление их в зависимости от типов разряда; расщепление фронта волны и образование предвестников [1-4]. Существенно, что в области плазменной оболочки и в области низкотемпературной плазмы двигателя газ становится колебательно неравновесным за счёт неупругих столкновений электронов плазмы с молекулами газа. Поэтому исследование особенностей взаимодействия ударных волн в колебательно-неравновесном газе с источником энергии, поддерживающим колебательную неравновесность, позволит эффективно управлять потоками неравновесной плазмы на крыле и в камере сгорания, что является актуальной задачей, в том числе для развития плазменной аэродинамики и ее приложений.

Как показано в [5-7], в средах со стационарно поддерживаемой неравновесностью изменение структуры слабой ударной волны может быть вызвано существенно новыми акустическими свойствами подобных сред, обусловленными знакопеременностью коэффициентов второй вязкости, дисперсии и газодинамической нелинейно-

Дмитрий Игоревич Завершинский, аспирант, каф. физики. Владимир Георгиевич Макарян (к.ф.-м.н.), доцент, каф. физики. Денис Петрович Порфиръев, ассистент, каф. физики. 
сти.

В настоящей работе численно исследовано взаимодействие ударной волны с областью стационарно неравновесного газа. Показано, что при проникновении в область неравновесности фронт ударной волны расщепляется с образованием тепловой и ударной волн, распространяющихся в область неравновесности.

Исходная система уравнений газодинамики включает уравнения непрерывности, Навье-Стокса, состояния газа и переноса тепла $[9,10]$ :

$$
\begin{aligned}
& \frac{d \rho}{d t}+\rho \frac{\partial u}{\partial x}=0, \\
& \rho \frac{d u}{d t}=-\frac{\partial P}{\partial x}+\frac{4}{3} \eta \frac{\partial^{2} u}{\partial x^{2}}, \\
& P=\rho T / m, \\
& C_{V \infty} \frac{d T}{d t}+\frac{d E_{K}}{d t}-\frac{T}{\rho} \frac{d \rho}{d t}=Q-I+\chi \frac{\partial^{2} T}{\partial x^{2}}+\frac{3 \eta m}{4 \rho}\left(\frac{\partial u}{\partial x}\right)^{2},
\end{aligned}
$$

где $d / d t=\partial / \partial t+u \partial / \partial x ; m-$ средняя молекулярная масса; $u-$ скорость газа; $T, \rho$, $P$ - температура (в энергетических единицах), плотность и давление среды; $C_{V \infty}-$ замороженная теплоемкость при постоянном объёме; $\eta, \chi$ - коэффициенты сдвиговой вязкости и теплопроводности газа. Систему (1)-(4) дополним уравнением релаксации внутренних степеней свободы. Для неравновесного возбуждения колебательных состояний молекул можно применить следующую модель релаксации:

$$
\frac{d E}{d t}=\frac{E_{e}-E}{\tau(T, \rho)}+Q,
$$

где $T_{k}$ - энергия колебательного кванта; $E$ - колебательная энергия в расчёте на одну молекулу, $E_{e}=T_{k} /\left(\exp \left(T_{k} / T\right)-1\right)$ - её равновесное значение (значение при равенстве стационарных колебательной и поступательной температур $T_{V}=T_{0}$ ); $\tau(T, \rho)=B \exp (b / \sqrt[3]{T}) / \rho \sqrt{T}$ - время колебательной релаксации согласно модели Ландау-Теллера [10]; $B$ и $b$-постоянные коэффициенты; $Q$ - мощность внешнего источника накачки (на одну молекулу), необходимая для поддержания неравновесности $E>E_{e} ; I$ - мощность теплоотвода в расчёте на одну молекулу.

Введём безразмерные переменные $\rho^{\prime}=\rho / \rho_{0}, u^{\prime}=u / c_{\infty}, P^{\prime}=P / P_{0}, T^{\prime}=T / T_{0}$, $S=Q \tau_{0} / T_{0}, E^{\prime}=E / T_{0}, E_{e}^{\prime}=E_{e} / T_{0}, \tau^{\prime}=\tau / \tau_{0}, t^{\prime}=t / \tau_{0}, x^{\prime}=x /\left(c_{\infty} \tau_{0}\right)$, где $\rho_{0}$, $P_{0}, T_{0}$ - невозмущенные значения плотности, давления и температуры (температура в энергетических единицах $) ; \tau_{0}=\tau\left(T_{0}, \rho_{0}\right), c_{\infty}=\sqrt{\gamma_{\infty} P_{0} / \rho_{0}}-$ высокочастотная скорость звука, $\gamma_{\infty}$ - высокочастотный показатель адиабаты; $S$ - степень неравновесности среды. После исключения давления из уравнения (2) с помощью уравнения состояния (3) и производных $d E / d t$ и $d \rho / d t$ из уравнения (4) с помощью уравнений (1) и (5) система уравнений (1)-(5) запишется так:

$$
\begin{aligned}
& \frac{\partial \rho^{\prime}}{\partial t^{\prime}}=-u^{\prime} \frac{\partial \rho^{\prime}}{\partial x^{\prime}}-\rho^{\prime} \frac{\partial u^{\prime}}{\partial x^{\prime}} \\
& \frac{\partial u^{\prime}}{\partial t^{\prime}}=-u^{\prime} \frac{\partial u^{\prime}}{\partial x^{\prime}}-\frac{1}{\gamma_{\infty}}\left(\frac{\partial T^{\prime}}{\partial x^{\prime}}+\frac{T^{\prime}}{\rho^{\prime}} \frac{\partial \rho^{\prime}}{\partial x^{\prime}}\right)+\frac{4 \eta}{3 \rho^{\prime} \rho_{0} \tau_{0} c_{\infty}^{2}} \frac{\partial^{2} u^{\prime}}{\partial x^{\prime 2}} \\
& \frac{\partial T^{\prime}}{\partial t^{\prime}}=-u^{\prime} \frac{\partial T^{\prime}}{\partial x^{\prime}}-\frac{1}{C_{V \infty}}\left(\frac{E_{e}^{\prime}-E^{\prime}}{\tau^{\prime}\left(\rho^{\prime}, T^{\prime}\right)}+S+T^{\prime} \frac{\partial u^{\prime}}{\partial x^{\prime}}-\frac{\chi}{\tau_{0} c_{\infty}^{2}} \frac{\partial^{2} T^{\prime}}{\partial x^{\prime 2}}-\right. \\
& \left.\frac{\partial E^{\prime}}{\partial t^{\prime}}=-u^{\prime} \frac{\partial E^{\prime}}{\partial x^{\prime}}+\frac{E_{e}^{\prime}-E^{\prime}}{\rho^{\prime} \rho_{0} \tau_{0} c_{\infty}^{2}}\left(\frac{\partial u^{\prime}}{\partial x^{\prime}}\right)^{2}\right),
\end{aligned}
$$




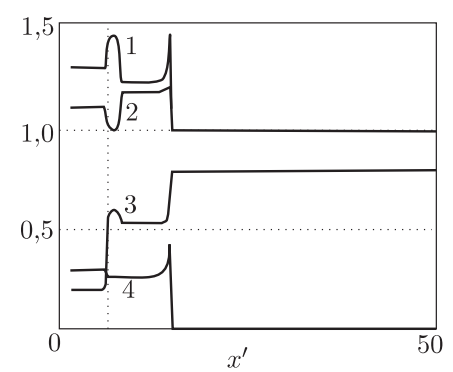

a

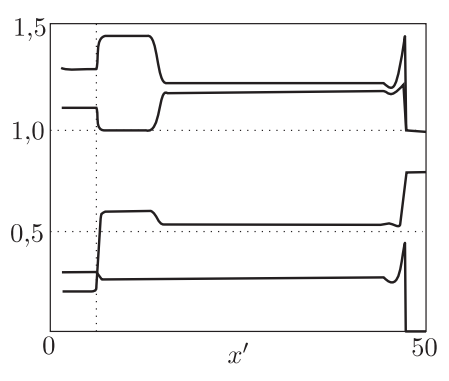

б

Результат численного моделирования проникновения ударной волны в колебательно-неравновесном газе в область со стационарной неравновесностью для двух моментов времени: a) $t^{\prime}=10$ и б) $t^{\prime}=35 ; 1-$ безразмерная плотность $\rho^{\prime} ; 2-$ безразмерная температура $T^{\prime} ; 3-$ безразмерная колебательная энергия $E^{\prime} ; 4$ - безразмерная скорость газа $u^{\prime}$

Для численного решения системы уравнений (6) пространственные производные аппроксимировались пятиточечными разностными функциями четвертого порядка точности:

$$
\frac{\partial \rho^{\prime}}{\partial x^{\prime}}=\frac{-\rho_{i+2}^{\prime}+8 \rho_{i+1}^{\prime}-8 \rho_{i-1}^{\prime}+\rho_{i-2}^{\prime}}{12 h}+\mathrm{O}\left(h^{4}\right)
$$

(аналогично для $\partial u^{\prime} / \partial x^{\prime}, \partial T^{\prime} / \partial x^{\prime}$ и $\left.\partial E^{\prime} / \partial x^{\prime}\right)$ и

$$
\frac{\partial^{2} u^{\prime}}{\partial x^{\prime 2}}=\frac{-u_{i+2}^{\prime}+16 u_{i+1}^{\prime}-30 u_{i}^{\prime}+16 u_{i-1}^{\prime}-u_{i-2}^{\prime}}{12 h^{2}}+\mathrm{O}\left(h^{4}\right)
$$

(аналогично для $\partial^{2} T^{\prime} / \partial x^{\prime 2}$ ). Здесь $h$ - величина шага сетки по пространственной координате. В результате система дифференциальных уравнений в частных производных (6) сводилась к системе обыкновенных дифференциальных уравнений для сеточных функций с производными первого порядка по времени. Интегрирование по времени полученной таким образом системы уравнений велось методом РунгеКутта 4-го порядка [10].

Результаты численного моделирования проникновения ударной волны в область стационарной неравновесности газа показаны на рисунке. Здесь начальная ударная волна распространяется в положительном направлении оси $x^{\prime}$. Прошедшая в неравновесную область ударная волна трансформируется в структуру двух возможных типов: с плавным убыванием плотности за ударным скачком (см. рис.) и структуру с плавным нарастанием плотности за ударным скачком. Подробно эти типы ударных волн исследованы в работах $[5-7,10-12]$. Хорошо видно расщепление фронта ударной волны на входе в область неравновесности (при $x^{\prime}>0$ ) с образованием тепловой волны охлаждения на границе области. Тепловая волна, возникающая на границе области неравновесности, распространяется внутрь этой области со скоростью потока газа. Она представляет собой область, в которой происходит плавное изменение плотности газа при неизменном давлении. Такое явление качественно совпадает с экспериментально наблюдавшимся расщеплением фронта ударной волны [13] при её проникновении в область плазмы тлеющего разряда.

Работа частично поддержана грантом Минобнауки РФ № 2.560.2011 в рамках государственных заданий высшим учебным заведениям на 2012 год и грантами ФЦП «Научные и научно-педагогические кадры инновационной России» на 2009-2013 гг. № 14.740.11.0999 и № 14.B37.21.0767. 


\section{БИБЛИОГРАФИЧЕСКИЙ СПИСОК}

1. Климов А.И., Коблов А.Н., Мишин Г.И., Серов Ю. Л., Явор И. П. Распространение ударных волн в нестационарном тлеющем разряде // Писъма в ЖТФ, 1989. Т. 15, № 20. C. 31-36. [Klimov A.I., Koblov A. N., Mishin G. I., Serov Yu. L., Yavor I. P. Shock Wave Propagation in transient Glow Discharge Plasmas // Pis'ma Zhurn. Tekhn. Fiz., 1989. Vol. 15, no. 20. Pp. 31-36].

2. Быстров С. А., Иванов В. И., Шугаев Ф. В. Распространение плоской ударной волны в слабоионизованной плазме // Физ. плазмы, 1989. Т. 15, №5. С. 558-562. [Bystrov S. A., Ivanov V.I., Shugaev F. V. Plane shock wave propagation in weakly ionized plasma// Fiz. Plazmy, 1989. Vol. 15, no. 5. Pp. 558-562].

3. Гридин А.Ю., Климов А.И., Молевич Н.Е. Распространение ударных волн в плазме тлеющего разряда // ЖTФ, 1993. Т. 63, №3. С. 157-162; англ. пер.: Gridin A. Yu., Klimov A. I., Molevich N.E. Propagation of shock waves in the plasma of a glow discharge // Tech. Phys., 1993. Vol. 38, no. 3. Pp. 238-240.

4. Гридин А.Ю., Климов А.И. Структура ударной волны в неравновесной плазме (выделение энергии, запасенной в разрядной плазме за ударной волной) // Xuм. физикa, 1993. T.12, №3. C. 363-365. [Gridin A. Yu., Klimov A. I. Shock wave structure in nonequilibrium plasma// Khim. Fiz., 1993. Vol.12, no. 3. Pp. 363-365].

5. Macheret S. O., Ionikh Yu.Z., Chernysheva N. V., Yalin A. P., Martinelli L., Miles R.B. Shock wave propagation and dispersion in glow discharge plasmas // Phys. Fluids, 2001. Vol. 13, no. 9, 2693. 13 pp.

6. Molevich N.E., Klimov A.I., Makaryan V.G. Influence of thermodynamical nonequilibrium on acoustical properties of gases // Int. J. aeroacoustics, 2005. Vol.4, no. 3-4. Pp. 373-384.

7. Макарян В.Г., Молевич Н.Е. Структура газодинамического возмущения в термодинамически неравновесной среде с экспоненциальной моделью релаксации // Изв. РАН. Mех. жидк. и газа, 2004. № 5. С. 181-191; англ. пер.: Makaryan V. G., Molevich N. E. Structure of a gasdynamic disturbance in a thermodynamically nonequilibrium medium with a power-law relaxation model // Fluid Dynamics, 2004. Vol.39, no. 5.

8. Макарян В.Г., Молевич Н.Е. Новые стационарные структуры в акустически активной среде // Писъма в ЖКТФ, 2003. Т. 29, №18. С. 11-15; англ. пер. Makaryan V. G., Molevich N. E. New stationary structures in an acoustically active medium// Tech. Phys. Lett, 2003. Vol. 29, no. 9. Pp. 752-753.

9. Макарян В. Г., Молевич Н. Е. Слабые ударные волны в неравновесных средах с отрицательной дисперсией // ЖKTФ, 2005. Т. 75, №6. С. 13-18; англ. пер.: Makaryan V. G., Molevich N.E. Weak shock waves in negative-dispersion nonequilibrium media// Tech. Phys., 2005. Vol.50, no. 6. Pp. 685-691.

10. Ландау Л.Д., Лифшич Е. М. Курс теоретической физики. Т. 6: Гидродинамика. М.: Наука, 1986. 736 с.; англ. пер.: Landau L. D. Lifshitz E. M. Course of Theoretical Physics. Vol. 6: Fluid Mechanics. New York: Pergamon, 1987. 552 pp.

11. Зелъдович Я. Б., Райзер Ю. П. Физика ударных волн и высокотемпературных гидродинамических явлений. М.: Наука, 1966. 688 с.; англ. пер.: Zel'dovich Ya. B., Raizer Yu.P. Physics of Shock Waves and High-Temperature Hydrodynamic Phenomena. New York: Academic, 1967. 994 pp.

12. Самарский A. A. Введение в численные методы. М.: Наука, 1982. 272 с. [Samarskiy A. A. An introduction to numerical methods. Moscow: Nauka, 1982. 272 pp.]

13. Makaryan V. G., Molevich N. E. Stationary shock waves in nonequilibrium media // Plasma Sources Sci. Technol., 2007. Vol.16, no.1. Pp. 124-131.

Поступила в редакцию 26/VII/2012;

в окончательном варианте - 21/VIII/2012. 
MSC: 76L05

INTERACTION OF SHOCK WAVES WITH AREA OF THE NON-EQUILIBRIUM IN VIBRATIONALLY EXCITED GAS

\section{I. Zavershinsky, V. G. Makaryan, D. P. Porfirev}

S. P. Korolyov Samara State Aerospace University

(National Research University),

34, Moskovskoe sh., Samara, 443086, Russia.

E-mail: vmak@rambler.ru

On the basis of numerical solution of the equations of gas dynamics of non-equilibrium medium the penetration of a shock wave in the area of non-equilibrium gas was investigated. The splitting of the shock wave front in a shock and heat waves was observed, it qualitatively coincides with the experimental results, obtained by A.I. Klimov.

Key words: shock wave, non-equilibrium state, vibrationally excited gas, shock adiabat, heat wave.

Original article submitted 26/VII/2012; revision submitted 21/VIII/2012.

Dmitriy I. Zavershinsky, Postgraduate Student, Dept. of Physics. Vladimir G. Makaryan (Ph. D. (Math. \& Phys.)), Associate Professor, Dept. of Physics. Denis P. Porfirev, Assistant, Dept. of Physics. 